\title{
Gut permeability and mucosal inflammation: bad, good or context dependent
}

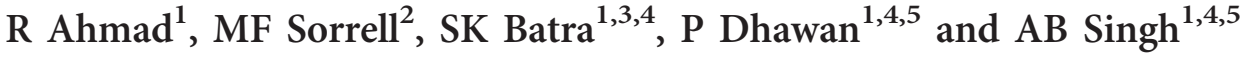

Inflammatory bowel disease (IBD) is a multifactorial disease. A breach in the mucosal barrier, otherwise known as "leaky gut," is alleged to promote mucosal inflammation by intensifying immune activation. However, interaction between the luminal antigen and mucosal immune system is necessary to maintain mucosal homeostasis. Furthermore, manipulations leading to deregulated gut permeability have resulted in susceptibility in mice to colitis as well as to creating adaptive immunity. These findings implicate a complex but dynamic association between mucosal permeability and immune homeostasis; however, they also emphasize that compromised gut permeability alone may not be sufficient to induce colitis. Emerging evidence further supports the role(s) of proteins associated with the mucosal barrier in epithelial injury and repair: manipulations of associated proteins also modified epithelial differentiation, proliferation, and apoptosis. Taken together, the role of gut permeability and proteins associated in regulating mucosal inflammatory diseases appears to be more complex than previously thought. Herein, we review outcomes from recent mouse models where gut permeability was altered by direct and indirect effects of manipulating mucosal barrier-associated proteins, to highlight the significance of mucosal permeability and the non-barrier-related roles of these proteins in regulating chronic mucosal inflammatory conditions.

\section{INTRODUCTION}

The gastrointestinal epithelium forms the body's largest interface with the external environment. It effectively provides a barrier that selectively limits permeation of luminal toxins and antigens through the mucosa, yet allows absorption of nutrients and water. ${ }^{1-3}$ To create this selective barrier, epithelial cells use both the transcellular and paracellular transport mechanisms. $^{3-5}$ However, it is the paracellular pathway, regulated by the tight junction (TJ), that is responsible for the greatest degree of apical cell-cell adhesion, ${ }^{6,7}$ and which has received the most attention for its role in regulating mucosal permeability under normal and pathological conditions. ${ }^{8-10}$ The perception that a breach in the mucosal barrier incites mucosal inflammation is supported by the physical location of the intestinal epithelium, interposed as it is between the luminal contents and the mucosal surface (Figure 1). The groundbreaking finding that mice expressing dominant-negative $\mathrm{N}$-cadherin, an adherent junction protein, develop spontaneous (not induced using chemicals or infections known to induce colitis in mice) inflammation in the gut epithelium further supports the key role of cell-to-cell adhesion, especially in its capacity to contribute to overall mucosal barrier function in regulating mucosal immune homeostasis. ${ }^{11}$ However, the discovery by Tsukita et al. ${ }^{12}$ in 1998 suggests that TJs, chiefly responsible for regulating paracellular permeability, are formed primarily by the newly identified claudin family of proteins. Claudin proteins are small $(20-27 \mathrm{kDa})$ transmembrane proteins expressed in tissue- and cell-specific manner ${ }^{12,13}$ with a total of 27 claudin family members having been identified to date. ${ }^{13}$ Studies investigating the status of claudins and other TJ-associated proteins in human biopsy specimen from chronic inflammatory conditions (Inflammatory Bowel Disease (IBD) $)^{14}$ have demonstrated differential changes in their total cellular content and/or distribution (see Table 1). These findings have led to the postulation that deregulated claudin expression helps to promote mucosal

${ }^{1}$ Department of Biochemistry and Molecular Biology, University of Nebraska Medical Center, 985870 Nebraska Medical Center, Omaha, Nebraska, USA. ${ }^{2}$ Department of Internal Medicine, Omaha, Nebraska, USA. ${ }^{3}$ Eppley Institute for Research in Cancer and Allied Diseases, Omaha, Nebraska, USA. ${ }^{4}$ Fred and Pamela Buffett Cancer Center, University of Nebraska Medical Center, Omaha, Nebraska USA and ${ }^{5}$ VA Nebraska-Western lowa Health Care System, Omaha, Nebraska USA. Correspondence: AB Singh (amar.singh@unmc.edu) 


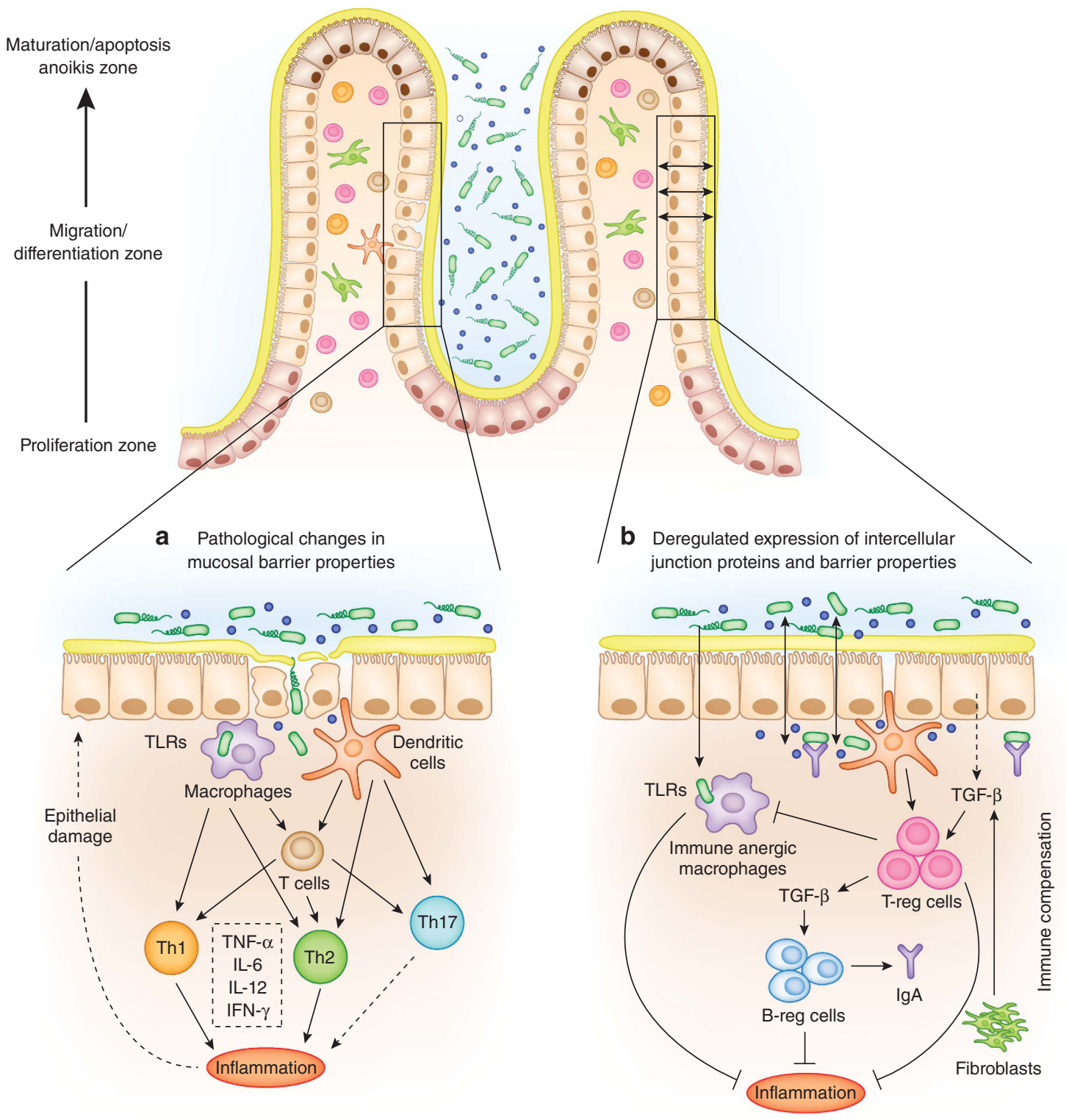

Figure 1 The intestinal epithelium constitutes a physical and biochemical barrier to separate effectively the luminal antigens and mucosal immune system. (a) A pathological breach in the mucosal barrier induces hyperactivation of the mucosal immune system and chronic inflammation. (b) However, a leaky gut due to opening of the tight junctions alone may not be sufficient to induce mucosal inflammation, but rather can induce adaptive immune response due to the heightened immune system. The ultimate outcome may depend on potential non-junctional effect/s of the barrier-associated protein/ s modified under inflammatory conditions.

inflammation. However, outcome from investigations using mice genetically manipulated for proteins integral or associated with the TJ have not been consistent and have resulted in diverse effects including immune adaptation and tolerance (summarized in Table 2). ${ }^{15,16}$ The compensatory immune mechanisms then act to protect against the development of colitis, even when there is increased gut permeability. ${ }^{15,16}$ Further, the non-tight-junctional roles of TJ-integral and associated proteins in regulating epithelial injury and repair have been identified. ${ }^{15,17,18}$ It is therefore important that we appreciate the comprehensive perspective of the role that $\mathrm{TJ}$ proteins may have in regulating immune homeostasis and epithelial injury, as well as their role in the repair of mucosal inflammation and associated diseases. In this review, we have discussed the historical viewpoint, and used current mouse models of colitis to highlight the complex but dynamic nature of the disease mechanism.

\section{THE TJ AND GUT PERMEABILITY}

The TJ (also referred to as zonula occludens) is the most apical cell-to-cell adhesion between neighboring epithelial and endothelial cells. ${ }^{1,12}$ As the name implies, TJ acts as a physical barrier whose function is to prevent free passage of ions and small solutes through the space between two interacting cells. 
Table 1 The review of the changes in tight junction-integral or -associated protein expression in inflammatory bowel disease and associated mouse models

\begin{tabular}{|c|c|c|c|c|c|}
\hline \multirow[t]{2}{*}{ Tight junction-integral/-associated proteins } & \multicolumn{2}{|c|}{ Inflammatory bowel disease } & \multicolumn{3}{|c|}{ Mouse models of colitis } \\
\hline & Crohn's disease & Ulcerative colitis & DSS & TNBS & Citrobacter rodentium \\
\hline Claudin-1 & $\uparrow^{49}$ & $\uparrow 93$ & $\uparrow^{94}$ & $\downarrow^{95}$ & No change ${ }^{96}$ \\
\hline Claudin-2 & $\uparrow^{49}$ & $\uparrow 49,97-99$ & $\uparrow^{94} \downarrow^{15}$ & $\uparrow^{100} \downarrow^{101}$ & Not known \\
\hline Claudin-3 & $\downarrow^{97,102}$ & $\downarrow^{97,102}$ & $\downarrow^{94}$ & $\downarrow^{103}$ & Delocalization 96,104 \\
\hline Claudin-4 & $\downarrow^{97,105}$ & $\downarrow^{97,105}$ & $\downarrow 106$ & Not known & $\downarrow^{107}$ \\
\hline Claudin-5 & $\downarrow^{98}$ & $\leftrightarrow^{98}$ & $\downarrow^{94}$ & No change & No change 96 \\
\hline Claudin-7 & $\leftrightarrow{ }^{105}$ & $\downarrow^{105}$ & $\downarrow^{94}$ & No change & Not known \\
\hline Claudin-8 & $\downarrow^{98}$ & $\leftrightarrow 98$ & $\downarrow^{94}$ & $\downarrow^{103}$ & Not known \\
\hline Claudin-12 & $\uparrow 108$ & $\leftrightarrow{ }^{108}$ & $\downarrow$ (rat) ${ }^{109}$ & Not known & Not known \\
\hline Claudin-18 & $\leftrightarrow^{110}$ & $\uparrow 110$ & Not known & Not known & Not known \\
\hline Occludin & $\downarrow^{98}$ & $\downarrow^{111}$ & $\downarrow^{106}$ & $\downarrow^{95}$ & Delocalization 104 \\
\hline Zo-1 & $\downarrow^{112}$ & $\leftrightarrow{ }^{112}$ & $\downarrow^{106}$ & $\downarrow^{95}$ & Delocalization 104 \\
\hline JAM-A & $\downarrow^{111}$ & $\downarrow^{113}$ & $\downarrow^{113}$ & $\downarrow^{114}$ & Delocalization \\
\hline
\end{tabular}

Abbreviations: DSS, dextran sodium sulfate; TNBS, trinitrobenzene sulfonic acid.

Nevertheless, it is not an impervious barrier, but rather selectively regulates what passes through the paracellular space. For this reason, TJs are considered to have a pivotal role in the regulation of intestinal permeability and diffusion of ions and molecules across the epithelial luminal surface. ${ }^{19-21}$ The TJ also serves as an intracellular hub where myriad of proteins reside alone or in a complex; these proteins have been demonstrated to be involved in adaptor, signaling, and cell polarity functions. $^{22,23}$ However, the core structure and function of the TJ depends on $\sim 50$ discrete membrane-integral and -associated proteins located between the apical and lateral regions of the polarized epithelial cells. ${ }^{24,25}$ These TJ proteins include ${ }^{1}$ integral and transmembrane proteins, including the claudins, occludin, and junctional adhesion molecules (JAM), which extend into the intercellular spaces to regulate the gate function, and ${ }^{2}$ cytoskeletal linker proteins, such as cingulin, ZO-1, ZO-2, and $\mathrm{ZO}-3$, which anchor TJ-integral membrane proteins to cell cytoskeleton. $^{12,26,27}$ Owing to its physical location and associated barrier properties, several lines of evidence support a direct link between TJ-constituent protein impairment and intestinal inflammation; however, a cause and effect relationship remains largely undefined, largely due to the complexity of mucosal barrier structure and function.

\section{THE HISTORICAL PERSPECTIVE}

Initial clinical findings that the intestinal epithelium of IBD patients is more permeable to tracer molecules known to permeate through the paracellular space $^{28-31}$ led to the hypothesis that an uncontrolled mucosal barrier may help promote IBD pathogenesis. Subsequent observations confirmed that there were ultrastructural changes in the junctional complexes between intestinal epithelial cells in IBD patient biopsy specimens and in related animal models of colitis. ${ }^{32,33}$ This hypothesis is supported by observations that the first- degree relatives of Crohn's disease (CD) patients are at increased risk of developing IBD and some of these relatives also demonstrate mucosal leakiness. ${ }^{34-36}$ Further complementing this hypothesis are the observations that hepatic and intestinal permeability markedly increases for transcellular (and paracellular) tracers such as horseradish peroxidase and lanthanum in animals subjected to experimental colitis. ${ }^{37}$ These observations neither confirmed nor denied a role for the mucosal barrier deregulation in initiating or promoting IBD, but simply supported a corroboration between mucosal leakiness and mucosal inflammatory conditions. Moreover, studies using animal models where gut permeability is altered because of the direct or indirect genetic or pharmacological manipulations have demonstrated diverse effects on mucosal immune homeostasis and inflammation, including immune adaptation and resistance to colitis. To grasp the clinical significance of this complex association, it is therefore necessary to review studies directly aimed at understanding it.

\section{Mice expressing dominant-negative $\mathrm{N}$-cadherin develop phenotype similar to IBD}

This study possibly provided the first experimental in vivo evidence that manipulating mucosal epithelial cell-to-cell adhesion can modulate mucosal barrier properties and that it promotes inflammation. This study was based on the hypothesis that cadherins mediate cell-to-cell adhesion and that they are essential for normal epithelial cell function and development. Therefore, interference with their function may lead to inflammatory conditions. In this study, embryonic stem cells were transfected with a dominant-negative N-cadherin mutant (NCAD delta) under the control of the Villin promoter, which is active primarily in intestinal epithelial cells, and then were introduced into C57BL/6 mouse blastocysts. Analysis of adult chimeric mice revealed robust expression of NCAD delta 
Table 2 Mouse models where tight junction-integral or -associated proteins have been genetically manipulated to assess impact on mucosal homeostasis and susceptibility to experimental colitis

\begin{tabular}{|c|c|c|c|c|c|}
\hline \multirow{2}{*}{$\begin{array}{l}\text { Cytoskeletal } \\
\text { proteins }\end{array}$} & \multicolumn{2}{|r|}{ Mouse models } & \multirow{2}{*}{$\begin{array}{l}\text { Associated } \\
\text { functions }\end{array}$} & \multirow{2}{*}{\multicolumn{2}{|c|}{$\begin{array}{l}\text { Potential implication in gastroin- } \\
\text { testinal pathophysiology }\end{array}$}} \\
\hline & Genotype & Phenotype & & & \\
\hline Claudin-1 & $\begin{array}{l}\text { Villin promoter-driven } \\
\text { overexpression }\end{array}$ & $\begin{array}{l}\text { Susceptible to experimentally induced } \\
\text { colitis }^{17,65,115}\end{array}$ & $\begin{array}{l}\text { Proliferation } \\
\text { Migration } \\
\text { Coreceptor } \\
\text { For hepatitis } \\
\text { C virus }\end{array}$ & $\uparrow$ & $\begin{array}{l}\text { Colon cancer promotion, coreceptor } \\
\text { for hepatitis } \mathrm{C} \text {, helps in antigen } \\
\text { sampling by dendritic cells }\end{array}$ \\
\hline Claudin-2 & $\begin{array}{l}\text { Villin promoter-driven } \\
\text { overexpression or } \\
\text { homozygous-knockout }\end{array}$ & $\begin{array}{l}\text { Increased gut permeability and immune com- } \\
\text { pensation Resistance to experimentally induced } \\
\text { colitis in overexpressing mice } \\
\text { Knockout mice are susceptible to experimentally } \\
\text { induced colitis }{ }^{15,48,53}\end{array}$ & $\begin{array}{l}\text { Proliferation } \\
\text { Migration }\end{array}$ & $\uparrow$ & Colon cancer promotion \\
\hline Claudin-7 & $\begin{array}{l}\text { Homozygous-knockout } \\
\text { or Villin-cre-mediated } \\
\text { knockout }\end{array}$ & $\begin{array}{l}\text { Increased gut permeability, spontaneous } \\
\text { mucosal inflammation } 63,66,116\end{array}$ & $\begin{array}{l}\text { Proliferation } \\
\text { Migration } \\
\text { Cell-matrix } \\
\text { adhesion }\end{array}$ & $\begin{array}{l}\downarrow \\
\uparrow\end{array}$ & Colon cancer suppressive \\
\hline JAM-A & Homozygous knockout & $\begin{array}{l}\text { Increased gut permeability; immune compen- } \\
\text { sation; resistance to DSS-colitis-induced } \\
\text { epithelial injury }^{16,113}\end{array}$ & $\begin{array}{l}\text { Proliferation } \\
\text { Migration }\end{array}$ & $\downarrow$ & $\begin{array}{l}\text { Promotes epithelial injury repair in } \\
\text { mice subjected to DSS-colitis }\end{array}$ \\
\hline Occludin & Homozygous knockout & Chronic gastritis ${ }^{40,117}$ & Proliferation & $\downarrow$ & $\begin{array}{l}\text { Decreased expression in IBD and } \\
\text { cancer }\end{array}$ \\
\hline $\begin{array}{l}\text { Myosin light chain } \\
\text { kinase }\end{array}$ & $\begin{array}{l}\text { Villin promoter-driven } \\
\text { overexpression }\end{array}$ & $\begin{array}{l}\text { Increased mucosal permeability; susceptible to } \\
\text { experimentally induced colitis }{ }^{10,41}\end{array}$ & Migration & $\downarrow$ & Induces diarrhea \\
\hline $\begin{array}{l}\text { Dominant-nega- } \\
\text { tive } \mathrm{N} \text {-cadherin } \\
\text { mutant (delta) }\end{array}$ & $\begin{array}{l}\text { Villin promoter-driven } \\
\text { overexpression }\end{array}$ & Spontaneous mucosal inflammation ${ }^{11}$ & $\begin{array}{l}\text { Proliferation } \\
\text { Migration } \\
\text { Cell } \\
\text { adhesion }\end{array}$ & $\uparrow$ & Colon cancer promotion \\
\hline P120-catenin & $\begin{array}{l}\text { Villin-cre-mediated } \\
\text { knockout }\end{array}$ & Spontaneous mucosal inflammation ${ }^{39}$ & $\begin{array}{l}\text { Proliferation } \\
\text { Migration } \\
\text { Cell } \\
\text { Adhesion }\end{array}$ & $\begin{array}{l}\uparrow \\
\downarrow\end{array}$ & Colon cancer promotion \\
\hline
\end{tabular}

Abbreviation: DSS, dextran sodium sulfate.

$\leftrightarrow$ No change.

along the entire crypt-villus axis, with the presence of spontaneous mucosal inflammation. However, these pathological manifestations were not exclusive of the modulations of epithelial homeostasis: NCAD delta expression perturbed proliferation, migration, and death programs in crypts, also leading to adenoma production. ${ }^{11}$ A similar phenotype was later described after manipulation of p120 catenin, a cytoplasmic binding partner that directly regulates cadherin stability. ${ }^{38}$ The p120 conditional knockout (KO) mice die young (within 21 days of their birth) and demonstrate progressive mucosal erosion and terminal bleeding, similar to what is observed in the dominant-negative cadherin mouse model of colitis and associated cancer, as described above. However, the observed phenotype in these mice was again not a simple ramification of mucosal barrier deregulation, but was the result of a conglomerate of defects in epithelial homeostasis, including epithelial cell apoptosis and barrier function irregularities. $^{39}$ The outcome of these studies reinforces the notion that the intestinal epithelium is essential for separating luminal antigens from mucosal immune components, and that its disruption by altered proliferation/apoptosis ratio can result in immune activation and inflammation (Figure 1). It is however unfortunate that strict determinations of the effects of these genetic changes on TJ-structure and mucosal barrier function were not carried out in these studies.

\section{Occludin KO mice develop chronic inflammation and hyperplasia despite normal mucosal barrier properties} The occludin $\mathrm{KO}$ mice represented the first in vivo experimental strategy to directly manipulate a TJ protein. ${ }^{40}$ Of note, gross deregulation of the cellular distribution of occludin, both intracellular and junction-bound occludin, was a predominant finding in a CD-3-induced mouse model of diarrhea. ${ }^{41}$ Here expression and/or cellular distribution of all claudin proteins, including claudin-2 and JAM-A, remained largely unaltered. ${ }^{41}$ Also of note, occludin-null mice were born in expected Mendelian ratios with no gross phenotype, although they did show significant postnatal growth retardation. ${ }^{40}$ Moreover, occludin-null males appeared infertile and did not produce litters when crossed with the wild-type (WT) females. On the other hand, the occludin-null females produced litters normally when mated with WT males but they did not suckle 
them. Irrespective, the TJ did not appear to be affected morphologically in these mice (of either sex) and the barrier function of the intestinal epithelium (small and large intestine), based on electrophysiological measurements, also appeared normal. However, several histological abnormalities were found in these mice, abnormalities that spanned several tissues and included chronic inflammation and hyperplasia of the gastric epithelium, calcification in the brain, testicular atrophy, loss of cytoplasmic granules in the striated duct cells of the salivary gland, and thinning of compact bone. ${ }^{40}$ In subsequent studies, Edelbium and co-workers, ${ }^{42}$ used occludin-null mice to further demonstrate defective accumulation and migration of intraepithelial $\gamma \delta$ lymphocytes within their intraepithelial compartment (vs. WT littermate mice). These observations stressed the complexity of the role that specific TJ-constituent proteins may have in regulating epithelial and immune homeostasis.

\section{Epithelial MLCK-dependent barrier dysfunction mediates T-cell activation-induced diarrhea in vivo}

Cytoskeletal contraction due to myosin II (regulatory)-light chain (MLC) phosphorylation is necessary for barrier disruption and in vitro inhibition of myosin light chain kinase (MLCK) restores barrier function following cytokine treatment. ${ }^{43}$ In a mouse model of T-cell-induced acute diarrhea, epithelial barrier dysfunction was associated with an abrupt increase in phospho (P)-MLC expression. However, observed barrier defects and associated diarrhea even in these mice did not result in changes in claudin-1, -2, -3 , and -4 expression and cellular distributions, but rather correlated with the rearrangement of cellular distribution of occludin, and to some extent with JAM proteins. ${ }^{41}$ Further studies also demonstrated that MLCK activation triggers caveolin-1dependent endocytosis of occludin to effect structural and functional TJ regulation. ${ }^{44}$ In subsequent studies, transgenic mice expressing constitutively active MLCK (CA-MLCK), specifically within the intestinal epithelial cells, demonstrated significant gut permeability in the jejunum (using bovine serum albumin) and the colon (using creatinine and sucralose); however, it did not develop spontaneous inflammation. ${ }^{10}$ Claudin-1, occludin, ZO-1, and JAM surface expression remained unaltered in these transgenic mice (vs. WT mice). Nonetheless, these mice harbored activated mucosal immune components as demonstrated by increased numbers of lamina propria $\mathrm{CD} 4(+)$ lymphocytes, redistribution of CD11c $(+)$ cells, increased production of the proinflammatory cytokines interferon- $\gamma$ and tumor necrosis factor- $\alpha$ (TNF- $\alpha$ ), and expression of epithelial major histocompatibility complex class-I. ${ }^{10}$ Also, when challenged with $\mathrm{CD} 4+\mathrm{CD} 45+\mathrm{Rb}$ (hi) lymphocytes, these mice experienced diarrhea and demonstrated increased susceptibility to colitis. ${ }^{10}$ These detailed studies further highlight the complexity of the mucosal barrier properties and reveal that alterations in cytoskeletal proteins and TJ-integral and -associated proteins can affect mucosal permeability as well as epithelial cell homeostasis.

\section{A transient breach in the epithelial barrier leads to the resistance to colitis}

This study was the first direct attempt to understand how physiologic breaks in the mucosal barrier, which must occur for homeostatic regulatory T cells to develop (Figure 1), may differ from the pathological mucosal barrier defects widely implicated in promoting inflammation of the underlying lamina propria. Here two different pharmacological agents known to induce gut permeability (ethanol and AT1002, a Vibrio cholerae zonula occludens toxin hexapeptide) were used to induce transient increase in gut permeability in mice before their exposure to experimental colitis. It was unexpected that the mice subjected to the transient increase in gut permeability were resistant to colitis induced by the administration of trinitrobenzene sulfonic acid. Further analysis suggested the role of transforming growth factor- $\beta$-dependent, preferential induction of T-regulatory cells in this immune adaptation. Of note, development of these cells required the presence of an intact microbiota and the activity of $\operatorname{CD} 11 \mathrm{c}(+)$ dendritic cells, along with innate immune factors operating through Tolllike receptor- $2{ }^{45}$ Thus, this study for the first time provided definitive evidence that the effect of an increase in gut permeability can be dependent on context. The outcome suggested that a "transient and limited increase in gut permeability" compared with the "unregulated breach of the gut barrier function due to the TJ deregulation and epithelial cell apoptosis" can rather lead to a dominant regulatory immune response to gut microbiota antigens and to a net anti-inflammatory effect.

\section{Compromised intestinal epithelial barrier due to intestinal epithelial-specific loss of JAM-A induces adaptive immune compensation that protects from colitis}

The findings in mice manipulated for MLCK or occludin expression differed from those obtained in mice lacking the JAM-A (encoded by F11r). ${ }^{16}$ However, the phenotype presented by JAM-A-null mice mirrored the phenotype presented by mice subjected to the transient breach in the barrier function before their exposure to acute colitis, to a great extent. ${ }^{18}$ In brief, JAM-A-null mice exhibited enhanced intestinal epithelial permeability, bacterial translocation and elevated colonic lymphocytes, but did not develop spontaneous colitis. ${ }^{16}$ To examine whether adaptive immune compensation in response to the increased gut permeability in these mice may have affected the overall phenotype, investigators examined the susceptibility of F11r $(-/-) \operatorname{Rag} 1(-/-)$ mice to acute colitis. Although contribution of the adaptive immunity in $\mathrm{F} 11 \mathrm{r}(+/$ $+) \operatorname{Rag} 1(-/-)$ mice was observed at negligible levels, $\operatorname{F} 11 \mathrm{r}(-/-) \operatorname{Rag} 1(-/-)$ mice exhibited increased microbiota-dependent colitis. Further investigations using elimination of the T-cell subsets and cytokine analyses revealed a protective role for the transforming growth factor- $\beta$-producing CD4 $(+)$ T cells in F11r $(-/-)$ mice. Moreover, loss of JAM-A resulted in elevated mucosal and serum immunoglobulin $\mathrm{A}$ that was dependent on CD4 $(+)$ T cells and tumor growth factor- $\beta$. The absence of immunoglobulin $\mathrm{A}$ in $\mathrm{F} 11 \mathrm{r}(+/+) \operatorname{Igha}(-/-)$ 
mice did not affect disease, whereas $\operatorname{F} 11 \mathrm{r}(-/-) \operatorname{Igha}(-/-)$ mice displayed markedly increased susceptibility to acute, injury-induced colitis. ${ }^{16}$ Notably, JAM-A also helps regulate intestinal epithelial cell ability to multiply and migrate, which may be of critical consideration in explaining the overall phenotype in these mice. ${ }^{46}$ The data from these mice, similar to those subjected to a transient increase in gut permeability, suggest that under conditions of compromised gut permeability, there are adaptive immune components activated in response to the hyperactivated immune system, and that these immune compensatory mechanisms may help provide resistance to colitis.

\section{Targeted and constitutive colonic claudin-2 expression renders resistance to epithelial injury, induces immune suppression, and protects from colitis}

The claudin family of transmembrane proteins are integral to TJ structure and function. ${ }^{12,26,47}$ Among the claudin family, claudin-2 is unique because it is expressed predominantly in epithelia known to be relatively less tight. ${ }^{48}$ Studies using biopsy samples from IBD patients have demonstrated that the expression of claudin-2 is highly upregulated during IBD. Because of the known association of claudin-2 with epithelial leakiness/permeability, increased claudin-2 expression has been suggested to promote mucosal inflammation. ${ }^{48,49}$ However, claudin-2 also serves other diverse functions including paracellular passage of cations (including sodium and calcium), cell proliferation and autophagy, and loss of claudin-2 along with claudin-15, yet another TJ protein implicated in paracellular $\mathrm{Na}^{+}$passage, results in premature death because of malnourishment in mice. ${ }^{48,50-52}$ However, the precise role of claudin-2 in regulating colonic homeostasis remains unclear. It was interesting to find that Villin-claudin-2 transgenic (CL-2TG) mice, generated in our laboratory, not only possessed a leakier but also a longer gut (vs. littermate control mice). Despite the leaky colon, as determined by electrophysiological measurements and presence of rectally administered FITC-dextran into blood circulation, $\mathrm{Cl}-2 \mathrm{TG}$ mice demonstrated resistance against acute or chronic colitis induced by administering the DSS in drinking water. ${ }^{15}$ Claudin-2 expression increased colonocyte proliferation and provided protection against colitis-induced colonocyte death in a phosphatidylinositol-4,5-bisphosphate 3 kinase/Bcl2-dependent manner. Moreover, $\mathrm{Cl}$-2TG mice demonstrated marked suppression of the colitis-induced immune activation and associated signaling, suggesting development of immune adaptation. Similarly, colon from the naive $\mathrm{Cl}-2 \mathrm{TG}$ mice harbored significantly increased number of regulatory $\mathrm{T}$ cells $(\mathrm{CD} 4(+)$ Foxp3 $(+)$ cells) than WT littermates. Also, macrophages isolated from $\mathrm{Cl}-2 \mathrm{TG}$ mouse colon exhibited immune anergy (failed to respond to the activation using proinflammatory cytokines) (Figure 2). These important immunosuppressive changes were associated with increased synthesis of the immunoregulatory cytokine transforming growth factor$\beta$ by colonic epithelial cells in Cl-2TG mice compared with WT littermates (Figure 2). ${ }^{15}$ The inverse findings by Nishida et al. ${ }^{53}$ using claudin-2-null mice, suggest that the loss of claudin-2 in colonic epithelium enhances susceptibility to the DSS-colitis complemented these findings. ${ }^{53}$ They not only revealed a critical, albeit complex, role of claudin-2 in intestinal homeostasis, regulating epithelial permeability, inflammation, and proliferation but also suggested a potential adaptive function for the increased claudin-2 expression in IBD patients. Obviously, further study is needed to accept or refute this proposition, and investigations are currently underway in our laboratory.

\section{Inflammation and disruption of the mucosal architecture in claudin-7-deficient mice}

The claudin family members are developmentally regulated and expressed in a tissue-specific manner. ${ }^{54-56}$ Interestingly, one of the claudin family proteins, claudin-7, when expressed in intestinal epithelium demonstrates a prominent basolateral membrane distribution compared with other claudins that localize primarily to the apical TJs in the intestinal epithelium. ${ }^{57}$ Also of note, integrins have an important role in regulating the mucosal epithelial and immune homeostasis. ${ }^{58-61}$ Moreover, ours studies and those of other laboratories suggest potential interdependence among $\beta 1$-integrin and claudin protein expression, which further suggests shared roles in regulating mammalian homeostasis. ${ }^{62-64}$ Recent studies using the wholebody or mucosal epithelial-specific deletion of claudin-7 support such a postulation. Moreover, these mice demonstrate a complex gastrointestinal phenotype involving mucosal inflammation, also associated with remarkable dysregulation of the mucosal epithelial homeostasis. ${ }^{63}$ It is important to note here that the TJ structure in claudin-7-null mice remained largely unaltered. Permeability assays suggested specific alteration in the paracellular passes for only small organic solutes. ${ }^{63}$ Overall, the authors suggested that potential non-TJal functions of claudin-7, including maintenance of epithelial cell-matrix interactions and intestinal homeostasis, may be responsible for the phenotype observed in claudin-7-null mice.

\section{Claudin-1 regulates intestinal epithelial homeostasis through the modulation of Notch signaling}

In a series of studies in our and collaborating laboratories (although predominantly using colorectal cancer-originated tissue and cell lines), we have observed an intriguing inverse correlation between claudin-7 and claudin-1 expressions. ${ }^{65,66}$ This inverse correlation between these two proteins is interesting in that it exists even in the normal gut epithelium. ${ }^{17,66}$ Mice deleted of claudin-1 protein expression die premature owing to dehydration, suggesting a key function for this protein in regulating dermal barrier function. ${ }^{67}$ It is, however, intriguing to observe that in gut pathologies, especially related to the colon and including chronic inflammatory conditions, claudin-1 protein expression is markedly upregulated (and mislocalized). ${ }^{49}$ Furthermore, the constitutive expression of claudin-1 in gut epithelium in mice resulted in decreased claudin-7 expression and enhanced susceptibility to acute colitis. Most notably, acute colitis induced by the administration of DSS in these mice failed to resolve even when DSS was removed from the drinking water, and this was found in contrast to the complete recovery seen in WT littermate 
a

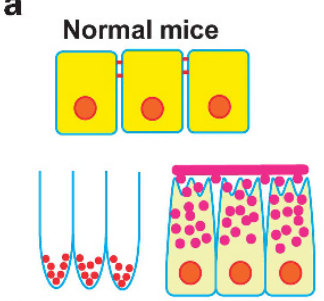

Normal proliferation and differentian
Claudin-1 TG mice

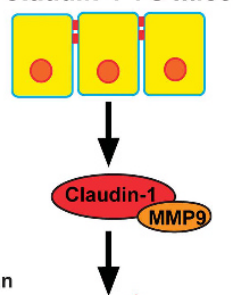

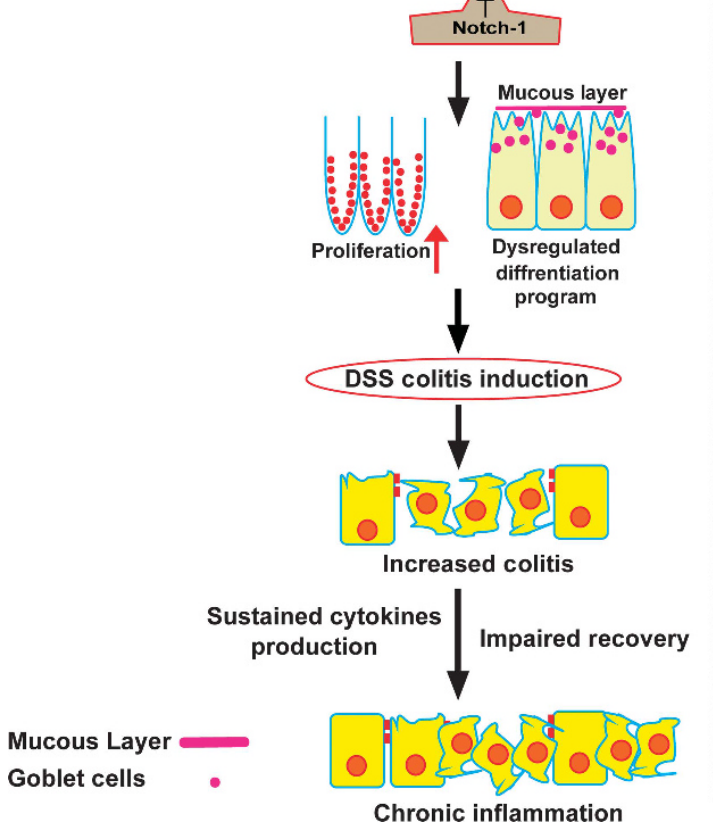

b

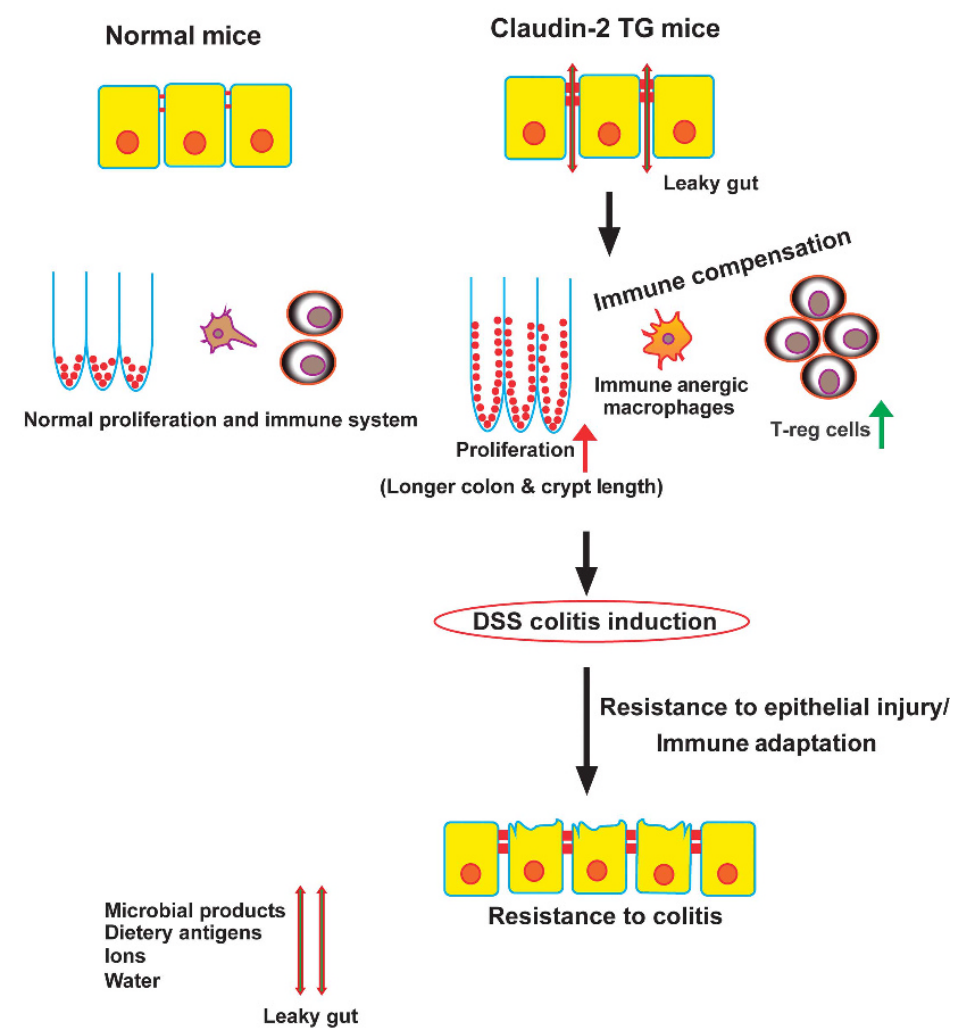

Figure 2 Alterations in tight junction proteins expression/cellular distribution may influence mucosal inflammatory conditions by modulating mucosal homeostasis and gut permeability. (a) Pathological (upregulated) expression of claudin-1 in mouse gut epithelium hyperactivates Notch-signaling to inhibit goblet cell/Mucin-2 synthesis, which in turn increases susceptibility to chronic inflammation. (b) Increased claudin-2 expression in mouse gut epithelium, similar to IBD patients, increases gut permeability and colonocyte proliferation. Presence of anergic macrophages and increased numbers of regulatory T cells (Treg cells) in the lamina propria of claudin-2 transgenic mice suggest immune compensation. As expected, these mice are resistant to experimentally induced colitis.

mice. ${ }^{68}$ Detailed analysis suggests a multipronged effect of the changes in intestinal claudin-1 expression, including mucosal barrier properties (mucin-2 expression) and epithelial homeostasis. These changes are especially important to note given the direct and indirect effects of alterations in claudin- 1 transgenic mice of normal colonocyte differentiation program, and mucin-2 expression in a matrix metalloproteinase-9/Notchdependent manner (Figure 2). ${ }^{17}$ These findings again highlighted the key function of specific TJ, integral or associated, proteins in regulating barrier function and epithelial homeostasis, which together could help explain altered expression of these proteins in IBD patients.

\section{WHAT HAVE WE LEARNED FROM MOUSE MODELS?}

While results from mouse models have failed to provide a definitive answer to settle the contributory role of TJ integral (and associated) proteins in regulating mucosal barrier dysfunction and inflammation, they have definitively clarified the critical role of these proteins in the maintenance of gut epithelial homeostasis. Dysfunction of intestinal epithelial cell polarity and function can trigger potent inflammatory responses within the gut, and related to this, mice deficient in Ap1m2, a master regulator of intestinal epithelial cell polarization, demonstrate chronic colitis. ${ }^{69}$ Furthermore, the overexpression of claudin-1 in the intestinal epithelium caused hyperactivation of Notch signaling and suppressed goblet cell differentiation and mucin-2 synthesis. ${ }^{17}$ These deregulations became more evident when claudin-1 TG mice were subjected to DSS-colitis and subsequent recovery resulted in persistent mucosal inflammation. ${ }^{17}$ These unique findings also highlighted a previously perceived, however poorly understood, interdependence between mucosal epithelium and overlying mucus layers, which collectively maintain the integrity of the mucosal barrier against luminal antigens (Figure 2). For this reason, the phenotype between claudin-1 TG mice and mucin-2 $\mathrm{KO}$ mice are similar, and they demonstrate robust colon tumor growth in the presence of APC mutation. ${ }^{68,70}$ The mucin protein family itself is highly diverse and contains membraneanchored or -secreted proteins with diverse functions, including regulation of the barrier function and epithelial cell 
homeostasis. ${ }^{70}$ Future investigations aimed at understanding the interdependence of mucins and claudin proteins may also provide an improved understanding of their shared roles in mucosal pathobiology. Contrary to claudin-1, which in normal colonic epithelial cells is expressed to a minimum (even though it is highly increased in colon cancer tissue and $c e l l s{ }^{65}$ ), the claudin-7 protein is highly expressed in the colonic epithelium. The findings that loss of claudin-7 expression induces sloughing of intestinal epithelial cells (claudin-7 KO mice), possibly by disrupting the complex between claudin-1, claudin-7 and $\beta 1$-integrin, highlights the previously underappreciated roles of TJ proteins in cell-matrix interactions. ${ }^{63}$ Moreover, mucosal inflammation in claudin-7 $\mathrm{KO}$ mice appears to be a product of epithelial cell instability and not necessarily the result of barrier deregulation, given that no apparent gut leakiness was documented at early time points in these mice. ${ }^{63}$ Considering its essential role in maintaining the differentiated colonic epithelium, claudin-7 expression is highly suppressed in transformed colonic epithelium and cancer tissues. ${ }^{71}$ Following similar trends, the active roles of both JAM-A and claudin-2 are implicated in regulating epithelial cell proliferation and mobility. ${ }^{15,18,48}$ The colon in claudin-2 TG mice is significantly longer than that of their littermates and their colonic epithelial proliferation is also upregulated. ${ }^{15}$ EGFR and Wnt signaling, two key regulators of colonic epithelial homeostasis (and tumorigenesis), stimulate colonic claudin-2 expression. ${ }^{48,72} \mathrm{We}$ further found that in colonic epithelial cells subjected to colitis causing chemical (DSS treatment), claudin-2 expression is sharply downregulated and that constitutive claudin-2 expression in these cells protected them from DSS-induced cell death. ${ }^{15}$ Of note, EGFR activation also protects from DSS-colitis. ${ }^{73-76}$ Taken together, an acceptable hypothesis appears to be that increased claudin-2 expression in inflamed mucosa of IBD patients may help promote epithelial injury and repair. Such a postulation has strong support based on the outcome from claudin-2 TG mice, which are resistant to colitis. ${ }^{15}$ However, a key function of the claudin-2 protein is also to serve as a paracellular transporter for $\mathrm{Na}^{+}$and $\mathrm{Ca}^{2+}$ ions, which may compete to pass through the claudin-2 pores. ${ }^{51,77}$ The role of these ions in the regulation of the normal intestinal physiology and pathobiology including mucosal inflammation is well recognized. ${ }^{78,79}$ One of the ways EGFR activation protects from experimental colitis in mice is by restituting $\mathrm{Na}^{+}$homeostasis. ${ }^{79}$ Thus, it will be interesting to examine the dynamic regulation of ion transport during inflammatory events, and whether there is a causal contribution of claudin-2 in such regulation. These examinations would require fine-tuned electrophysiological studies and precise analysis. Nevertheless, the contributory role of the TJ protein in mucosal barrier properties and pathobiology remains undeniable, but discerning their separate roles in barrier deregulation and epithelial morphogenesis appears to be a truly difficult task, at least given current technologies and model systems.

\section{EPITHELIAL INTEGRITY, BARRIER DYSFUNCTION, AND IMMUNE CYTOKINES: A TWO-WAY COMMUNICATION}

Increased mucosal permeability not only includes increased paracellular flux across the TJ but also through the spaces created by intestinal epithelial cell damage and death. Thus, we must discuss the potential regulation of epithelial integrity and barrier function under conditions of immune activation and inflammation. Inflammatory cytokines regulate TJ protein expression and function, as well as overall mucosal barrier properties. The most studied cytokine known to cause barrier dysfunction is TNF- $\alpha$. TNF- $\alpha$ treatment, as determined by freeze-fracture electron microscopy studies, decreases TJ strand number and complexity in HT29/B6 cells, while increasing the frequency of strand breaks. ${ }^{80}$ Among TJ proteins, TNF- $\alpha$ modulates the expression of many members where it upregulates claudin-2 expression, inhibits occludin promoter activity, and causes redistribution of ZO-1 and claudin- $1 .{ }^{81-83}$ Anti-TNF- $\alpha$ therapy restores gut barrier function in CD. However, TNF- $\alpha$ also helps regulate intestinal epithelial cell death and injury or repair. ${ }^{84}$ Additionally, interleukin-17 (IL-17) regulates claudin-1 and claudin-2 expression in T84 cells in MEK/ERK1/2-dependent manner. ${ }^{85}$ IL-15 can also regulate TJ formation in intestinal epithelial cells. However, IL-15-mediated upregulation of ZO-1 and ZO-2 expression was independent of the IL-2R $\beta$ subunit, whereas phosphorylation of occludin and enhanced membrane association of claudin-1 and claudin- 2 by IL- 15 required the presence of the IL-2R $\beta$ subunit. ${ }^{86,87}$ Similarly, IL-6 modulation of the claudin-2 gene induces intestinal epithelial TJ permeability through the JNK pathway. ${ }^{88}$ Similarly, IL-13 the T-helper type 2 cytokine and key effector of ulcerative colitis also affects intestinal epithelial cell TJ function as well as apoptosis, ${ }^{89}$ and was recently shown to regulate claudin-2 transcription in a signal transducers and activators of transcription-dependent manner. ${ }^{90}$ Overall, IBD represents a conglomerate of complex changes in mucosal cytokine/chemokine production. The net effect of these changes on mucosal barrier properties and disease outcome can thus be expected to be the net balance of their pro- and antiinflammatory effects. ${ }^{91}$ Furthermore, growth factor signaling, which helps regulate colonic epithelial cell injury and repair, also regulates the expression of $\mathrm{TJ}$ protein expression in intestinal epithelial cells. ${ }^{92}$ Thus, it appears that dynamic crosstalk between epithelial and immune cell homeostasis maintains normal gut functioning, and that disruption of any of these factors seems capable of inducing conditions favoring an inflammatory environment. However, outcomes may also depend on genetic predisposition to inflammation. Nevertheless, these discussions in no way include the multifactorial nature of the disease processes encompassing IBD, and intentionally have not included potential implications of genetic predispositions, spontaneous immune deregulations, proteases, and microbiota, which are discussed in great detail in other great review articles. 


\section{CONCLUSION}

The mechanisms regulating intestinal epithelial and immune homeostasis are intricate and interdependent. Still, the etiology of IBD is complex and not yet fully understood: ongoing research will broaden the horizon of knowledge regarding the mechanisms underlying the pathobiology of chronic intestinal inflammation. It is hoped that the central role of the intestinal epithelium in this regulation will become more evident. The emerging data from mice manipulated for TJ-integral or associated proteins support the critical yet complex roles of these proteins in the regulation of intestinal epithelial homeostasis and mucosal permeability. It appears that the effects of gut permeability on immune activation and intestinal inflammation may vary, depending on the extent of mucosal barrier deregulation, and the context in which this occurs. Nonetheless, the aforementioned animal models, although extremely useful in investigating specific molecular mechanisms in the pathogenesis of ulcerative colitis, do not truly recapitulate disease as observed in patients, as IBD is a multifactorial disease and most clearly is not caused by a single mutation or defect in cellular or molecular pathways. Thus, going forward, it will be important to focus on dynamic changes observed in those proteins associated with mucosal barrier properties, rather than simply documenting terminal modifications as they are expressed in chronically inflamed mucosa. We will base our conclusions on this understanding about these proteins and their potential role in barrier deregulation and/or repair and regeneration properties. Our conclusions will both relate to and can have profound implications for disease prognosis. These findings may also provide an opportunity for novel and targeted therapeutic uses, although more studies are needed in this direction to clarify cause and effect associations.

\section{ACKNOWLEDGMENTS}

We thank all the researchers who have spent their time and effort in trying to understand the complexity of this issue, and we extend our apologies if we could not accommodate a particular work in this review because of space constraints. This work was supported by VA-merit BX002086 (to P.D.), DK088902 (to A.B.S.) and BX002761 (to A.B.S.).

\section{DISCLOSURE}

The authors declared no conflict of interest.

c 2017 Society for Mucosal Immunology

\section{REFERENCES}

1. Farquhar, M. G. \& Palade, G. E. Junctional complexes in various epithelia. J. Cell Biol. 17, 375-412 (1963).

2. Claude, P. \& Goodenough, D. A. Fracture faces of zonulae occludentes from "tight" and "leaky" epithelia. J. Cell Biol. 58, 390-400 (1973).

3. Powell, D. W. Barrier function of epithelia. Am. J. Physiol. 241, G275-G288 (1981).

4. Schneeberger, E. E. \& Lynch, R. D. Structure, function, and regulation of cellular tight junctions. Am. J. Physiol. 262 (Part 1), L647-L661 (1992).

5. Tang, V. W. \& Goodenough, D. A. Paracellular ion channel at the tight junction. Biophys. J. 84, 1660-1673 (2003).

6. Gumbiner, B. Structure, biochemistry, and assembly of epithelial tight junctions. Am. J. Physiol. 253 (Part 1), C749-C758 (1987).

7. Tsukita, S. \& Furuse, M. Pores in the wall: claudins constitute tight junction strands containing aqueous pores. J. Cell Biol. 149, 13-16 (2000).
8. Wyatt, J., Vogelsang, H., Hubl, W., Waldhoer, T. \& Lochs, H. Intestinal permeability and the prediction of relapse in Crohn's disease. Lancet 341, 1437-1439 (1993).

9. D'Inca, R. et al. Intestinal permeability test as a predictor of clinical course in Crohn's disease. Am. J. Gastroenterol. 94, 2956-2960 (1999).

10. Su, L. et al. Targeted epithelial tight junction dysfunction causes immune activation and contributes to development of experimental colitis. Gastroenterology 136, 551-563 (2009).

11. Hermiston, M. L. \& Gordon, J. I. Inflammatory bowel disease and adenomas in mice expressing a dominant negative $\mathrm{N}$-cadherin. Science 270, 1203-1207 (1995).

12. Furuse, M., Fujita, K., Hiiragi, T., Fujimoto, K. \& Tsukita, S. Claudin-1 and 2: novel integral membrane proteins localizing at tight junctions with no sequence similarity to occludin. J. Cell Biol. 141, 1539-1550 (1998).

13. Mineta, K. et al. Predicted expansion of the claudin multigene family. FEBS Lett. 585, 606-612 (2011).

14. Sartor, R. B. Current concepts of the etiology and pathogenesis of ulcerative colitis and Crohn's disease. Gastroenterol. Clin. N. Am. 24 475-507 (1995).

15. Ahmad, R. et al. Targeted colonic claudin-2 expression renders resistance to epithelial injury, induces immune suppression, and protects from colitis. Mucosal Immunol. 7, 1340-1353 (2014).

16. Khounlotham, M. et al. Compromised intestinal epithelial barrier induces adaptive immune compensation that protects from colitis. Immunity 37, 563-573 (2012).

17. Pope, J. L. et al. Claudin-1 regulates intestinal epithelial homeostasis through the modulation of Notch-signalling. Gut 63, 622-634 (2014).

18. Laukoetter, M. G. et al. JAM-A regulates permeability and inflammation in the intestine in vivo. J. Exp. Med. 204, 3067-3076 (2007).

19. Baumgart, D. C. \& Dignass, A. U. Intestinal barrier function. Curr. Opin. Clin. Nutr. Metab. Care 5, 685-694 (2002).

20. Berkes, J., Viswanathan, V. K., Savkovic, S. D. \& Hecht, G. Intestinal epithelial responses to enteric pathogens: effects on the tight junction barrier, ion transport, and inflammation. Gut 52, 439-451 (2003).

21. Mitic, L. L., Van Itallie, C. M. \& Anderson, J. M. Molecular physiology and pathophysiology of tight junctions I. Tight junction structure and function: lessons from mutant animals and proteins. Am. J. Physiol. Gastrointest. Liver Physiol 279, G250-G254 (2000).

22. Tsukita, S., Yamazaki, Y., Katsuno, T., Tamura, A. \& Tsukita, S. Tight junction-based epithelial microenvironment and cell proliferation. Oncogene 27, 6930-6938 (2008).

23. Matter, K. \& Balda, M. S. Signalling to and from tight junctions. Nat. Rev. Mol. Cell Biol. 4, 225-236 (2003).

24. Cereijido, M. et al. New diseases derived or associated with the tight junction. Arch. Med. Res. 38, 465-478 (2007).

25. Anderson, J. M., Van Itallie, C. M. \& Fanning, A. S. Setting up a selective barrier at the apical junction complex. Curr. Opin. Cell Biol. 16, 140-145 (2004).

26. Furuse, M. et al. Occludin: a novel integral membrane protein localizing at tight junctions. J. Cell Biol. 123 (6 Pt 2), 1777-1788 (1993).

27. Fanning, A. S., Jameson, B. J., Jesaitis, L. A. \& Anderson, J. M. The tight junction protein ZO-1 establishes a link between the transmembrane protein occludin and the actin cytoskeleton. J. Biol. Chem. 273, 29745-29753 (1998).

28. Hering, N. A., Fromm, M. \& Schulzke, J. D. Determinants of colonic barrier function in inflammatory bowel disease and potential therapeutics. J. Physiol. 590 (Part 5), 1035-1044 (2012).

29. Hollander, D. Crohn's disease-a permeability disorder of the tight junction?. Gut 29, 1621-1624 (1988).

30. Sundqvist, T., Magnusson, K. E., Sjodahl, R., Stjernstrom, I. \& Tagesson, C. Passage of molecules through the wall of the gastrointestinal tract. II. Application of low-molecular weight polyethyleneglycol and a deterministic mathematical model for determining intestinal permeability in man. Gut 21, 208-214 (1980).

31. Ukabam, S. O., Clamp, J. R. \& Cooper, B. T. Abnormal small intestinal permeability to sugars in patients with Crohn's disease of the terminal ileum and colon. Digestion 27, 70-74 (1983).

32. Marin, M. L., Greenstein, A. J., Geller, S. A., Gordon, R. E. \& Aufses, A. H. Jr. A freeze fracture study of Crohn's disease of the terminal ileum: 
changes in epithelial tight junction organization. Am. J. Gastroenterol. 78, 537-547 (1983).

33. Marin, M. L., Geller, S. A., Greenstein, A. J., Marin, R. H., Gordon, R. E. \& Aufses, A. H. Jr Ultrastructural pathology of Crohn's disease: correlated transmission electron microscopy, scanning electron microscopy, and freeze fracture studies. Am. J. Gastroenterol. 78, 355-364 (1983).

34. Turner, J. R. Intestinal mucosal barrier function in health and disease. Nat. Rev. Immunol. 9, 799-809 (2009).

35. Katz, K. D. et al. Intestinal permeability in patients with Crohn's disease and their healthy relatives. Gastroenterology 97, 927-931 (1989).

36. Buhner, S. et al. Genetic basis for increased intestinal permeability in families with Crohn's disease: role of CARD15 3020insC mutation?. Gut 55, 342-347 (2006).

37. Fries, W. etal. Experimental colitis increases small intestine permeability in the rat. Lab. Invest. 79, 49-57 (1999).

38. Ireton, R. C. et al. A novel role for p120 catenin in E-cadherin function. J. Cell Biol. 159, 465-476 (2002).

39. Smalley-Freed, W. G. et al. p120-catenin is essential for maintenance of barrier function and intestinal homeostasis in mice. J. Clin. Invest. 120, 1824-1835 (2010).

40. Saitou, M. et al. Complex phenotype of mice lacking occludin, a component of tight junction strands. Mol. Biol. Cell 11, 4131-4142 (2000).

41. Clayburgh, D. R. et al. Epithelial myosin light chain kinase-dependent barrier dysfunction mediates T cell activation-induced diarrhea in vivo. J. Clin. Invest. 115, 2702-2715 (2005).

42. Edelblum, K. L. et al. Dynamic migration of gammadelta intraepithelial lymphocytes requires occludin. Proc. Natl Acad. Sci. USA 109 (18), 7097-7102 (2012).

43. Zolotarevsky, Y. et al. A membrane-permeant peptide that inhibits MLC kinase restores barrier function in in vitro models of intestinal disease. Gastroenterology 123, 163-172 (2002).

44. Marchiando, A. M. et al. Caveolin-1-dependent occludin endocytosis is required for TNF-induced tight junction regulation in vivo. J. Cell Biol. 189, 111-126 (2010).

45. Boirivant, M. et al. A transient breach in the epithelial barrier leads to regulatory T-cell generation and resistance to experimental colitis. J. Cell Biol. 12, 1612-1623 e5 (2008).

46. Nava, P. et al. JAM-A regulates epithelial proliferation through Akt/betacatenin signalling. EMBO Rep. 12, 314-320 (2011).

47. Singh, A. B., Sharma, A. \& Dhawan, P. Claudin family of proteins and cancer: an overview. J. Oncol. 2010, 541957 (2010).

48. Dhawan, P. et al. Claudin-2 expression increases tumorigenicity of colon cancer cells: role of epidermal growth factor receptor activation. Oncogene 30, 3234-3247 (2011).

49. Weber, C. R., Nalle, S. C., Tretiakova, M., Rubin, D. T. \& Turner, J. R. Claudin-1 and claudin-2 expression is elevated in inflammatory bowel disease and may contribute to early neoplastic transformation. Lab. Invest. 88, 1110-1120 (2008).

50. Tamura, A. et al. Loss of claudin-15, but not claudin-2, causes $\mathrm{Na}^{+}$ deficiency and glucose malabsorption in mouse small intestine. Gastroenterology 140, 913-923 (2011).

51. Wada, M., Tamura, A., Takahashi, N. \& Tsukita, S. Loss of claudins 2 and 15 from mice causes defects in paracellular $\mathrm{Na}^{+}$flow and nutrient transport in gut and leads to death from malnutrition. Gastroenterology 144, 369-380 (2013).

52. Nighot, P. K., Hu, C. A. \& Ma, T. Y. Autophagy enhances intestinal epithelial tight junction barrier function by targeting claudin-2 protein degradation. J. Biol. Chem. 290, 7234-7246 (2015).

53. Nishida, M., Yoshida, M., Nishiumi, S., Furuse, M. \& Azuma, T. Claudin-2 regulates colorectal inflammation via myosin light chain kinase-dependent signaling. Dig. Dis. Sci. 58, 1546-1559 (2013).

54. Turksen, K. \& Troy, T. C. Claudin-6: a novel tight junction molecule is developmentally regulated in mouse embryonic epithelium. Dev. Dyn. 222, 292-300 (2001).

55. Patel, R. M., Myers, L. S., Kurundkar, A. R., Maheshwari, A., Nusrat, A. \& Lin, P. W. Probiotic bacteria induce maturation of intestinal claudin 3 expression and barrier function. Am. J. Pathol. 180, 626-635 (2012).
56. Lee, B., Kang, H. Y., Lee, D. O., Ahn, C. \& Jeung, EB Claudin-1 2, 4, and 5: comparison of expression levels and distribution in equine tissues. $J$ Vet Sci. 17, 445-451 (2016).

57. Rahner, C., Mitic, L. L. \& Anderson, J. M. Heterogeneity in expression and subcellular localization of claudins 2, 3, 4, and 5 in the rat liver, pancreas, and gut. Gastroenterology 120, 411-422 (2001).

58. Berlin, C. et al. Alpha 4 beta 7 integrin mediates lymphocyte binding to the mucosal vascular addressin MAdCAM-1. Cell 74, 185-195 (1993).

59. Wagner, N. et al. Critical role for beta7 integrins in formation of the gutassociated lymphoid tissue. Nature 382, 366-370 (1996).

60. Schon, M. P. et al. Mucosal T lymphocyte numbers are selectively reduced in integrin alpha $\mathrm{E}$ (CD103)-deficient mice. J. Immunol. 162, 6641-6649 (1999).

61. Mebius, R. E., Schadee-Eestermans, I. L. \& Weissman, I. L. MAdCAM-1 dependent colonization of developing lymph nodes involves a unique subset of CD4 + CD3 - hematolymphoid cells. Cell Adhes. Commun. 6, 97-103 (1998).

62. Tabaries, S. et al. Claudin-2 is selectively enriched in and promotes the formation of breast cancer liver metastases through engagement of integrin complexes. Oncogene 30, 1318-1328 (2011).

63. Ding, L. et al. Inflammation and disruption of the mucosal architecture in claudin-7-deficient mice. Gastroenterology 142 , 305-315 (2012).

64. Elias, B. C. et al. The integrin beta1 subunit regulates paracellular permeability of kidney proximal tubule cells. J. Biol. Chem. 289, 8532-8544 (2014).

65. Dhawan, P. et al. Claudin-1 regulates cellular transformation and metastatic behavior in colon cancer. J. Clin. Invest. 115, 1765-1776 (2005).

66. Bhat, A. A. et al. Claudin-7 expression induces mesenchymal to epithelial transformation (MET) to inhibit colon tumorigenesis. Oncogene 34, 4570-4580 (2015).

67. Furuse, M. et al. Claudin-based tight junctions are crucial for the mammalian epidermal barrier: a lesson from claudin-1-deficient mice. J. Cell Biol. 156, 1099-1111 (2002).

68. Pope, J. L., Ahmad, R., Bhat, A. A., Washington, M. K., Singh, A. B. \& Dhawan, P. Claudin-1 overexpression in intestinal epithelial cells enhances susceptibility to adenamatous polyposis coli-mediated colon tumorigenesis. Mol. Cancer 13, 167 (2014).

69. Takahashi, D. et al. The epithelia-specific membrane trafficking factor AP$1 \mathrm{~B}$ controls gut immune homeostasis in mice. Gastroenterology 141, 621-632 (2011).

70. Van der Sluis, M. etal. Muc2-deficient mice spontaneously develop colitis, indicating that MUC2 is critical for colonic protection. Gastroenterology 131, 117-129 (2006).

71. Bhat, A. A. et al. Claudin-7 expression induces mesenchymal to epithelial transformation (MET) to inhibit colon tumorigenesis. Oncogene 34, 4570-4580 (2014).

72. Mankertz, J., Hillenbrand, B., Tavalali, S., Huber, O., Fromm, M. \& Schulzke, J. D. Functional crosstalk between Wnt signaling and Cdxrelated transcriptional activation in the regulation of the claudin-2 promoter activity. Biochem. Biophys. Res. Commun. 314, 1001-1007 (2004).

73. Dube, P. E. et al. Epidermal growth factor receptor inhibits colitisassociated cancer in mice. J. Clin. Invest. 122, 2780-2792 (2012).

74. Brandl, K. et al. MyD88 signaling in nonhematopoietic cells protects mice against induced colitis by regulating specific EGF receptor ligands. Proc. Natl Acad. Sci. USA 107, 19967-19972 (2010).

75. Lu, N. et al. Activation of the epidermal growth factor receptor in macrophages regulates cytokine production and experimental colitis. J. Immunol. 192, 1013-1023 (2014).

76. Monticelli, L. A., Osborne, L. C., Noti, M., Tran, S. V., Zaiss, D. M. \& Artis, D. IL-33 promotes an innate immune pathway of intestinal tissue protection dependent on amphiregulin-EGFR interactions. Proc. Natl Acad. Sci. USA 112, 10762-10767 (2015).

77. Yu, A. S., Cheng, M. H. \& Coalson, R. D. Calcium inhibits paracellular sodium conductance through claudin-2 by competitive binding. J. Biol. Chem. 285, 37060-37069 (2010). 
78. Barkas, F., Liberopoulos, E., Kei, A. \& Elisaf, M. Electrolyte and acid-base disorders in inflammatory bowel disease. Ann. Gastroenterol. 26, 23-28 (2013).

79. McCole, D. F., Rogler, G., Varki, N. \& Barrett, K. E. Epidermal growth factor partially restores colonic ion transport responses in mouse models of chronic colitis. Gastroenterology 129, 591-608 (2005).

80. Schmitz, H. et al. Tumor necrosis factor-alpha (TNFalpha) regulates the epithelial barrier in the human intestinal cell line HT-29/B6. J. Cell Sci. 112 (Pt 1), 137-146 (1999).

81. Mankertz, J. et al. Expression from the human occludin promoter is affected by tumor necrosis factor alpha and interferon gamma. J. Cell Sci. 113 (Pt 11), 2085-2090 (2000).

82. Wang, F., Graham, W. V., Wang, Y., Witkowski, E. D., Schwarz, B. T. \& Turner, J. R. Interferon-gamma and tumor necrosis factor-alpha synergize to induce intestinal epithelial barrier dysfunction by up-regulating myosin light chain kinase expression. Am. J. Pathol. 166, 409-419 (2005).

83. Weber, C. R. et al. Epithelial myosin light chain kinase activation induces mucosal interleukin-13 expression to alter tight junction ion selectivity. J. Biol. Chem. 285, 12037-12046 (2010).

84. Hobbs, S. S. et al. TNF transactivation of EGFR stimulates cytoprotective COX-2 expression in gastrointestinal epithelial cells. Am. J. Physiol. Gastrointest. Liver Physiol. 301, G220-G229 (2011).

85. Kinugasa, T., Sakaguchi, T., Gu, X. \& Reinecker, H. C. Claudins regulate the intestinal barrier in response to immune mediators. Gastroenterology 118, 1001-1011 (2000).

86. Stevens, A. C. et al. Interleukin- 15 signals T84 colonic epithelial cells in the absence of the interleukin-2 receptor beta-chain. Am. J. Physiol. 272 (Part 1), G1201-G1208 (1997).

87. Nishiyama, R. et al. Interleukin-2 receptor beta subunit-dependent andindependent regulation of intestinal epithelial tight junctions. J. Biol. Chem. 276, 35571-35580 (2001).

88. Al-Sadi, R. et al. Interleukin-6 modulation of intestinal epithelial tight junction permeability is mediated by $\mathrm{JNK}$ pathway activation of claudin-2 gene. PLoS One 9, e85345 (2014).

89. Heller, F. et al. Interleukin-13 is the key effector Th2 cytokine in ulcerative colitis that affects epithelial tight junctions, apoptosis, and cell restitution. Gastroenterology 129, 550-564 (2005).

90. Rosen, M. J. et al. STAT6 deficiency ameliorates severity of oxazolone colitis by decreasing expression of claudin-2 and Th2-inducing cytokines. J. Immunol. 190, 1849-1858 (2013).

91. DiGuilio, K. M. et al. Sieving characteristics of cytokine- and peroxideinduced epithelial barrier leak: Inhibition by berberine. World J. Gastrointest. Pathophysiol. 7, 223-234 (2016).

92. Peterson, L. W. \& Artis, D. Intestinal epithelial cells: regulators of barrier function and immune homeostasis. Nat. Rev. Immunol. 14, 141-153 (2014).

93. Poritz, L. S., Harris, L. R. III, Kelly, A. A. \& Koltun, W. A. Increase in the tight junction protein claudin-1 in intestinal inflammation. Dig. Dis. Sci. 56, 2802-2809 (2011).

94. Xia, X. M., Wang, F. Y., Zhou, J., Hu, K. F., Li, S. W. \& Zou, B. B. CXCR4 antagonist AMD3100 modulates claudin expression and intestinal barrier function in experimental colitis. PLoS One 6, e27282 (2011).

95. Kang, G. D. \& Kim, D. H. Poncirin and its metabolite ponciretin attenuate colitis in mice by inhibiting LPS binding on TLR4 of macrophages and correcting Th17/Treg imbalance. J. Ethnopharmacol. 189, 175-185 (2016).

96. Guttman, J. A., Samji, F. N., Li, Y., Vogl, A. W. \& Finlay, B. B. Evidence that tight junctions are disrupted due to intimate bacterial contact and not inflammation during attaching and effacing pathogen infection in vivo. Infect. Immun. 74, 6075-6084 (2006).

97. Prasad, S. et al. Inflammatory processes have differential effects on claudins 2, 3 and 4 in colonic epithelial cells. Lab. Invest. 85, 1139-1162 (2005).
98. Zeissig, S. etal. Changes in expression and distribution of claudin 2, 5 and 8 lead to discontinuous tight junctions and barrier dysfunction in active Crohn's disease. Gut 56, 61-72 (2007).

99. Denizot, J. et al. Adherent-invasive Escherichia coli induce claudin-2 expression and barrier defect in CEABAC10 mice and Crohn's disease patients. Inflamm. Bowel Dis. 18, 294-304 (2012).

100. Liu, T. et al. Vitamin D treatment attenuates 2,4,6-trinitrobenzene sulphonic acid (TNBS)-induced colitis but not oxazolone-induced colitis. Sci. Rep. 6, 32889 (2016).

101. Liu, W. et al. Intestinal epithelial vitamin D receptor signaling inhibits experimental colitis. J Clin Invest 123, 3983-3996 (2013).

102. Lameris, A. L. et al. Expression profiling of claudins in the human gastrointestinal tract in health and during inflammatory bowel disease. Scand. J. Gastroenterol. 48, 58-69 (2013).

103. Li, Q. et al. Effect of $n-3$ polyunsaturated fatty acids on membrane microdomain localization of tight junction proteins in experimental colitis. FEBS J. 275, 411-420 (2008).

104. Gibson, D. L. et al. Toll-like receptor 2 plays a critical role in maintaining mucosal integrity during Citrobacter rodentium-induced colitis. Cell Microbiol. 10, 388-403 (2008).

105. Oshima, T., Miwa, H. \& Joh, T. Changes in the expression of claudins in active ulcerative colitis. J. Gastroenterol. Hepatol. 23 (Suppl 2), S146S150 (2008).

106. Mennigen, R. et al. Probiotic mixture VSL\#3 protects the epithelial barrier by maintaining tight junction protein expression and preventing apoptosis in a murine model of colitis. Am. J. Physiol. Gastrointest. Liver Physiol. 296, G1140-G1149 (2009).

107. Morampudi, V. et al. Vasoactive intestinal peptide prevents PKCepsiloninduced intestinal epithelial barrier disruption during EPEC infection. Am. J. Physiol. Gastrointest. Liver Physiol. 308, G389-G402 (2015).

108. Amasheh, S., Fromm, M. \& Gunzel, D. Claudins of intestine and nephron - a correlation of molecular tight junction structure and barrier function. Acta Physiol. (Oxf) 201, 133-140 (2011).

109. Arimura, Y., Nagaishi, K. \& Hosokawa, M. Dynamics of claudins expression in colitis and colitis-associated cancer in rat. Methods Mol. Biol. 762, 409-425 (2011).

110. Zwiers, A., Fuss, I. J., Leijen, S., Mulder, C. J., Kraal, G. \& Bouma, G. Increased expression of the tight junction molecule claudin-18 A1 in both experimental colitis and ulcerative colitis. Inflamm. Bowel Dis. 14, 1652-1659 (2008)

111. Kucharzik, T., Walsh, S. V., Chen, J., Parkos, C. A. \& Nusrat, A. Neutrophil transmigration in inflammatory bowel disease is associated with differential expression of epithelial intercellular junction proteins. Am. J. Pathol. 159, 2001-2009 (2001).

112. Mees, S. T. et al. Expression of tight and adherens junction proteins in ulcerative colitis associated colorectal carcinoma: upregulation of claudin-1, claudin-3, claudin-4, and beta-catenin. Int. J. Colorectal Dis. 24, 361-368 (2009).

113. Vetrano, S. et al. Unique role of junctional adhesion molecule-a in maintaining mucosal homeostasis in inflammatory bowel disease. Gastroenterology 135, 173-184 (2008).

114. Gerlach, K., McKenzie, A. N., Neurath, M. F. \& Weigmann, B. IL-9 regulates intestinal barrier function in experimental Tcell-mediated colitis. Tissue Barriers 3, e983777 (2015).

115. Evans, M. J. et al. Claudin- 1 is a hepatitis $C$ virus co-receptor required for a late step in entry. Nature 446, 801-805 (2007).

116. Tanaka, H., Takechi, M., Kiyonari, H., Shioi, G., Tamura, A. \& Tsukita, S. Intestinal deletion of Claudin-7 enhances paracellular organic solute flux and initiates colonic inflammation in mice. Gut 64, 1529-1538 (2015).

117. Osanai, M., Murata, M., Nishikiori, N., Chiba, H., Kojima, T. \& Sawada, N. Epigenetic silencing of occludin promotes tumorigenic and metastatic properties of cancer cells via modulations of unique sets of apoptosisassociated genes. Cancer Res. 66, 9125-9133 (2006). 